\title{
Discrete Distortion for Surface Meshes
}

\author{
Mohammed Mostefa Mesmoudi, Leila De Floriani, and Paola Magillo
}

Department of Computer Science, University of Genova

Via Dodecaneso n 35, 16146 Genova Italy

mmesmoudi@ac-creteil.fr,

\{deflo,magillo\}@disi.unige.it

http://www.disi.unige.it

\begin{abstract}
Discrete distortion for two- and three-dimensional combinatorial manifolds is a discrete alternative to Ricci curvature known for differentiable manifolds. Here, we show that distortion can be successfully used to estimate mean curvature at any point of a surface. We compare our approach with the continuous case and with a common discrete approximation of mean curvature, which depends on the area of the star of each vertex in the triangulated surface. This provides a new, area-independent, tool for curvature estimation and for morphological shape analysis. We illustrate our approach through experimental results showing the behavior of discrete distortion.
\end{abstract}

Keywords: Gaussian curvature, mean curvature, discrete curvature estimators, triangle meshes.

\section{Introduction}

Intrinsic properties of a surface, like genus, Euler-Poincaré characteristic, Betti numbers, orientability, serve to classify it topologically. Other properties, like tangent plane, normal vector at a point and the shape, serve to describe local geometry of the surface. Further properties, like first and second fundamental forms, principal curvature, Gaussian curvature, mean curvature, geodesic curvature, serve to study the surface from a metric point of view. In practical applications, we need to deal with discrete representations of surfaces, usually consisting of triangle meshes, called triangulated, or polyhedral surfaces. Metric properties of such surfaces have to be described in a discrete coherent way since they are usually known for smooth surfaces. From this point of view, many numerical methods are proposed in the literature, which often present problems in error control and estimation, and often have a high computational complexity.

Curvature plays a crucial role to understand the geometry and the topology of a surface. For analytic surfaces, the mathematical background is well developed 9], 16. We find, thus, notions of geodesic, normal, principal, mean, Gaussian and total curvatures. All of them give a precise description of the local geometric shape of a surface in any direction.

In the discrete case, Gaussian curvature is generally estimated, according to the Gauss-Bonnet theorem, by computing the total angle at a vertex and by 
dividing it by the area of its neighborhood (see, for instance, [17]). All existing methods have approximation errors and many of them suffer from the convergence problem due to the choice of the neighborhood. This means that the curvature values strongly depend on the area of the local neighborhood of a point, and diverge generally when the area goes to 0 (by refining the mesh for example). The scale of curvature values depends generally on the method used. Moreover, those methods do not give importance the (local) geometric shape of the surface, while curvature is strongly related to it in the smooth case. Concentrated curvature [1], known also as the angle deficit method, is based only on angles estimation, and, thus, has many advantages: curvature values have a natural meaning, are accurate and simple to compute. Furthermore, concentrated curvature describes the global topology of the surfaces via a discrete equivalent version of Gauss-Bonnet theorem generally known for the smooth case. On the other hand, concentrated curvature does not describe the local geometric shape of the surface.

Mean curvature generally serves to identify some local geometric characteristics of the surfaces, namely ridges and ravines. Moreover, the combination of mean curvature combined and Gaussian curvature identifies morphological characteristics of the surface, such as convex and concave regions. A local geometric classification of points based on the sign of both Gaussian and mean curvature is presented in [16.

In a recent work [12, the authors have introduced a new notion, called discrete distortion, which is a local discrete approach to Ricci curvature for three-dimensional combinatorial manifolds. Discrete distortion has promising applications in many scientific fields (medical imagery, particle physics, general relativity, engineering mechanics,...). For three-dimensional manifolds with boundary, discrete distortion provides a new tool to explore the local geometry of surfaces viewed as boundary components of the 3-manifold.

The purpose of this work is to show that discrete distortion can be used as a tool for estimating mean curvature for triangulated surfaces. Specifically, distortion can be used to explore the morphology of a surface and to identify some of its features, such as ridges, ravines, convex and concave regions.

The remainder of this paper is organized as follows. In Section 2, we discuss related work on curvature estimation. In Section 3, we review fundamental notions related to discrete Gaussian and mean curvature and some notions related to spherical geometry. In Section 4, we recall the notions of discrete vertex and bond distortions for surfaces, and some of their properties. In Section 5, we investigate relations between discrete distortion and mean curvature. In Section 6. we present experimental results illustrating the behavior of distortion, and we compare it with a widely used mean curvature estimator. In Section 7 , we draw some concluding remarks, and discuss our current and future work.

\section{Related Work}

Two major approaches exist in the literature to estimate curvature over triangulated surfaces: analytic methods and discrete methods [8]. Analytic approaches 
consist of interpolating over the vertices of the triangle mesh through smooth functions (generally quadratic functions) and of computing the curvature analytically on the resulting smooth approximation [7]. Analytic methods seem to perform quite well, but are computationally intensive, and, thus, not suitable for large-size triangulated surfaces.

Discrete approaches are numerous. They basically use discretization of derivatives of Gauss-Bonnet formula, or of Euler formula. Linearization of first and second derivatives is used in the first discrete approach to approximate, with some error estimates, curvature values [10]. Issues related to discrete approaches are error estimation and control, convergence problems over small areas or irregular meshes (see [8, [11, 13] for more details and discussion).

Concentrated curvature has been recently used by some authors [12] under the name of angle deflection and in [1] to define a discrete approximation of Gaussian curvature. In [11] a comparative study with the deficit angle method, mostly used in literature, has been performed. A purely combinatorial approach to concentrated curvature has been introduced in [3].

\section{Background Notions}

In this Section, we briefly review some notions related to discrete curvature and spherical geometry necessary for developing the material of this paper.

\subsection{Discrete Mean Curvature}

In computer graphics, smooth surfaces are approximated by discrete models, like clouds of points or triangle meshes. In mathematics, curvature is defined for smooth surfaces that are at least $C^{2}$ continuous. Hence, strictly speaking, curvature is not defined in the discrete case. In practice, techniques exist which provide curvature estimates for discrete surfaces. When the surface is represented by a triangle mesh, curvature at internal points of triangles has a null value. At points on triangulation edges, or at vertices, different methods exist to estimate the Gaussian and the mean curvatures. As discussed in Section 2, many problems exist for those methods which involve the area of a neighborhood of a point. A good, area-independent, alternative is presented in [11. This curvature estimator is called, originally in mathematics [18, concentrated curvature.

Concentrated curvature at a vertex $p$ is equal to the total curvature of a spherical cap approximating the surface, from its interior, around $p$. This quantity is expressed by means of the total angle at $p$. Thus, it is independent of the radius of the local neighborhood at $p$ and hence of its area. Moreover, it has been shown that it satisfies a discrete version of Gauss-Bonnet theorem. Based on the idea of angle estimation, discrete distortion has been introduced in [12 for three-dimensional combinatorial manifolds. Its restriction to boundary surfaces is the purpose of this work.

In the discrete case, mean curvature at a vertex $p$ of a polyhedral surface is usually defined [13] by

$$
|H|=\frac{1}{4|A|} \sum_{i=1}^{n}\left\|\overrightarrow{e_{i}}\right\|\left|\beta_{i}\right|
$$


where $|A|$ is the the area of the Voronoi or barycentric region around $p, e_{i}$ is one of the $n$ edges incident in $p$, and $\beta_{i}$ is the angle between the normal vectors of the two faces sharing edge $e_{i}$. If we denote with $\Theta_{i}$ the dihedral angle at $e_{i}$, then angle $\beta_{i}$ is the supplementary angle of $\Theta_{i}$, and Formula 1 becomes

$$
|H|=\frac{1}{4|A|} \sum_{i=1}^{n}\left\|\overrightarrow{e_{i}}\right\|\left|\pi-\Theta_{i}\right| .
$$

This formula can be justified as follows. Suppose that we want to approximate the surface at an edge $e$ by a cylindric cap of radius $r$ tangent to the surface from its interior at the faces adjacent to $e$. The total curvature of the circle arc generating the cap is equal to the absolute value of the supplementary angle $\beta$ of the dihedral angle $\Theta$ at $e$ (cf. [19] for a proof) and does not depend on the radius $r$. The curvature of any generating edge is null. Then the mean curvature at a point of the cylindrical cap is equal to $\frac{|\beta|+0}{2}$. Hence, the total mean curvature of the cylindrical cap is equal to $\frac{|\beta|}{2}$ multiplied by the length of the generating edge $e$. In general, the surrounding neighborhood of a vertex is taken to be the Voronoi area or the barycentric area around the vertex. Then only half of the incident edges of $p$ are considered. This explain Formula 2 .

However, there are two issues with the use of Formula 2 for a mean curvature estimator. The first issue is that its value depends on the length of the edges incident in vertex $v$, and thus it is area-dependent. The second issue is that such value is always positive since only absolute values are taken. This implies, for example, that the mean curvature of a saddle surface is always non-negative, which is clearly not true for analytic surfaces.

\subsection{Spherical Geometry}

Here, we briefly review some notions related to spherical geometry needed to introduce the notion of distortion.

Let $T$ be a tetrahedron in the Euclidean space $R^{3}$. We refer to Figure 1, Let $O$ be one of the four vertices of $T$. We consider the unit sphere centered at $O$, and the intersection points $A, B$, and $C$ of the supporting half-lines of the three edges of $T$ emanating from $O$. The trihedral angle, also called the solid angle, at $O$ corresponds to the area of the spherical triangle $A B C$ obtained from the intersection of the unit sphere centered at $O$ with the cone $\prec(O A B C)$. The trihedral angle at $O$ is given by the formula

$$
\widehat{A}+\widehat{B}+\widehat{C}-\pi
$$

where $\widehat{A}, \widehat{B}, \widehat{C}$, are the dihedral angles at vertices $A, B$ and $C$. This formula is known to be Girard's theorem [14.

The dihedral angle between two adjacent faces is the supplementary of the angle between their normal vectors. It can easily be proven that a dihedral angle, without loss of generality let us say the one formed by the two faces incident in the edge containing $A$, that we denote with $\widehat{A}$, can be obtained by the following relation:

$$
\cos (\widehat{A})=\frac{\cos a-\cos b \cos c}{\sin b \sin c}
$$


where $a, b$ and $c$ are respectively the angles at $O$ of the tetrahedron faces $O B C$, $O C A$ and $O A B$, i.e., the lengths of the corresponding spherical arcs on the unit sphere. Each of them can be obtained from the inner product of the corresponding vectors.

Other trigonometric relations are available for spherical triangles [15].

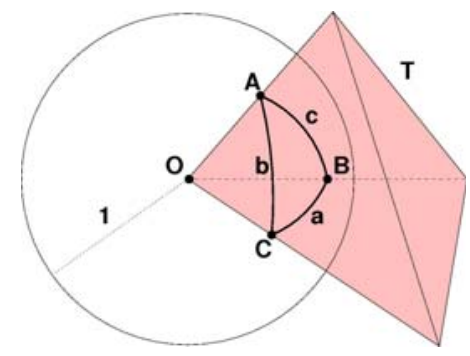

Fig. 1. Tetrahedron $T$, the unit sphere centered at vertex $O$ of $T$, the spherical triangle $A B C$, and the three spherical arcs $a, b, c$

\section{Discrete Distortion}

In this Section, we discuss the notion of distortion for triangulated surfaces with some of its properties (see [12] for more details).

Let $p$ be a vertex in a triangulated surface $\Sigma$. Vertex distortion at $p$ is defined by the quantity

$$
D(p)=2 \pi-S_{p},
$$

where $S_{p}$ is the solid angle at $p$.

Similarly for each edge $e$ of the mesh Bond distortion of edge $e$, is the quantity $\bar{D}(e)$ defined by

$$
\bar{D}(e)=\pi-\Theta_{e},
$$

where $\Theta_{e}$ is the dihedral angle at edge $e$.

Theorem 1. Vertex distortion at a vertex $p$ is the sum of all bond distortions around edges incident to $p$. We have

$$
D(p)=\sum_{e \in S t(p)}\left(\pi-\Theta_{e}\right),
$$

where St $(p)$ is the one-ring neighborhood of $p$.

Note that, for the interior points of the triangles, the distortion is null since the area of spherical caps are semi-Euclidean spheres. For a point in the interior of an edge $e$, the spherical cap is a sphere segment delimited by the two faces adjacent to $e$. Its area is equal to twice the dihedral angle $\Theta$ at $e$. Thus, distortion of such points is given by

$$
D(p)=2\left(\pi-\Theta_{e}\right)=2 \bar{D}(e) .
$$




\section{Discrete Distortion as a Mean Curvature Estimator}

Let $S$ be a smooth surface (at least $C^{2}$ ). Let $p$ be a point of $S$ and $S(p, r)$ be a sphere centered at $p$ of radius $r>0$. Surface $S$ cuts $S(p, r)$ into two parts. Let $A(p, r)$ be the area of the cap contained within the internal volume of $S$. For $r$ sufficiently small, the quantity

$$
C(p, r)=\frac{A(p, r)}{r^{2}}
$$

is the value of the Connolly function at $(p, r)$. This function has been introduced by Connolly in the seventies to study knobs and depressions of molecular shapes 44. In the smooth case, for small values of $r$, this quantity is equivalent to $2 \pi$ since the spherical cap converges to the half sphere located above the tangent plane. However, the convergence speed is proportional to the mean curvature as shown in the following Lemma:

Lemma 1 [4]. There exists a $C^{\infty}$ function $\epsilon$ defined on $S \times R^{+}$such that $\epsilon$ tends to 0 when $r$ goes to 0 and

$$
C(p, r)=2 \pi+\pi H(p) r+r^{2} \epsilon(p, r)
$$

where $H(p)$ is the mean curvature of $S$ at $p$.

In the discrete case, for each vertex $p \in S$, there exists $r_{0}$ such that the intersection of the spheres $(S(p, r))_{r<r_{0}}$ with surface $S$ is located within the 1-ring neighborhood $S t(p)$ of $p$ and the Connolly function is constant. Then $D(p)=2 \pi-C(p, r)$ for any $r \leq r_{0}$. Combining this idea with Lemma 1, Connolly function (and thus distortion) can be used to estimate mean curvature values as explained below.

Under the assumptions of Lemma 1, the quantity $D(p, r):=2 \pi-C(p, r)$ at $p$ is equal to

$$
D(p, r)=-\pi H(p) r-r^{2} \epsilon(p, r)
$$

Specifically, for a fixed $r$

$$
\operatorname{Grad}_{p}(D)=-\pi r \operatorname{Grad}_{p}(H)-r^{2} \operatorname{Grad}(\epsilon(p, r))
$$

Formulas 11 and 12 allow us to find those points $p$ for which $D(p, r)$ vanishes and the critical points of $D(p, r)$. Indeed, Formula 11 implies that, if $H(p)=0$, then the values of $D(p, r) / r$ are close to 0 for sufficiently small $r$. This means that points with a null mean curvature are located in regions for which $D(p, r) / r$, and, hence, $D(p, r)$ is sufficiently close to 0 . Conversely, if $D(p, r)=0$, then Formula 11 gives $H(p)=\frac{r}{\pi} \epsilon(p, r)$, and this quantity is close to 0 if $r$ is sufficiently small. Thus, points for which $D(p, r)$ vanishes are located in regions of small mean curvature.

Similar conclusions can be obtained from Formula [12, to find the critical points of $D(p, r)$ and $H(p)$ (i.e., points $p$ for which $\operatorname{Grad}_{p}(D)=0$ and point $p$ for which $\left.\operatorname{Grad}_{p}(H)=0\right)$. 


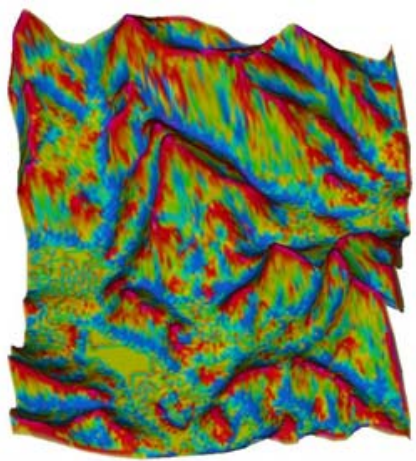

Distortion (Marcy)

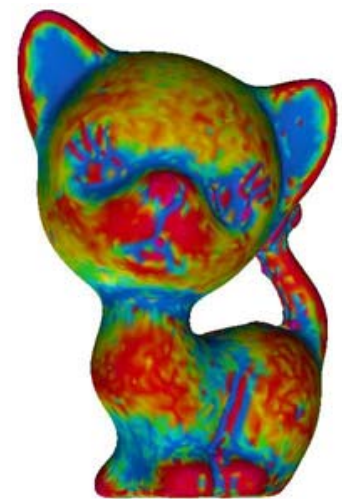

Distortion (Kitten)

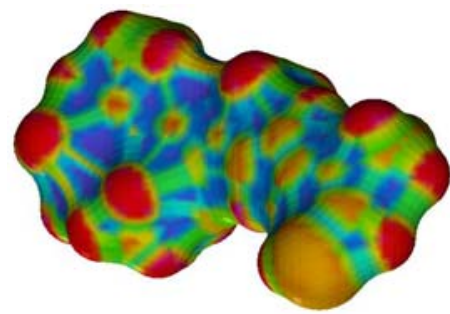

Distortion (Retinal)

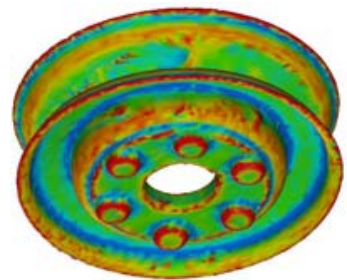

Distortion (Pulley)

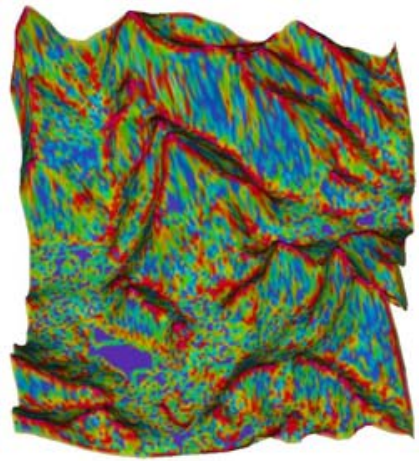

Mean (Marcy)

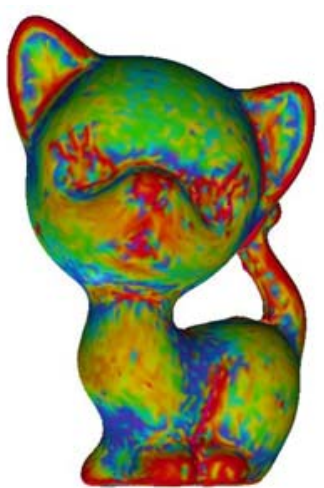

Mean (Kitten)

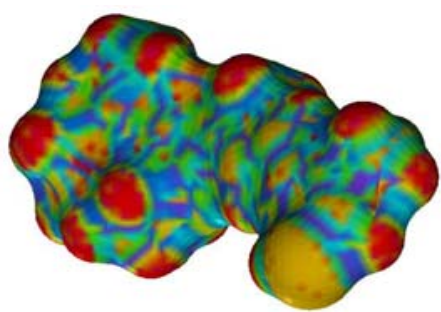

Mean (Retinal)

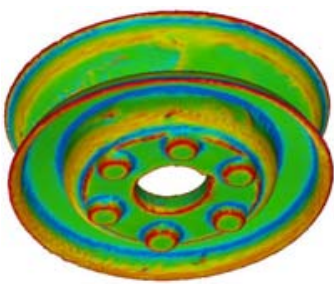

Mean (Pulley)

Fig. 2. Distortion and mean curvature on the four data sets 
In the discrete case, where values can be considered as null for values of $r$ below a certain threshold, theses properties allow localizing minimal surfaces (i.e., regions with null mean curvature), ravine and ridge regions by means of our discrete distortion. Furthermore, discrete distortion serves also to characterize convex and concave regions. Convex regions have positive distortion values, while concave regions have negative distortion values.

\section{Experimental Results}

We have implemented an algorithm that computes discrete distortion for surfaces. Here, we present experimental results to compare the behavior of discrete distortion and that of the mean curvature estimator expressed by Formula 2, We compare distortion and mean curvature on real data:

- Marcy (terrain, $128 \times 128$ vertices).

- Kitten (3D surface of a statue, 11039 vertices)

- Retinal (3D surface of a molecule, 3643 vertices)

- Pulley (3D surface of a mechanical piece, 25482 vertices)

We acknowledge Frederic Cazals for the Retinal mesh. The other three meshes are from the Aim at Shape Repository (http://shapes.aim-at-shape.net/).

In Figure 2 a rainbow-like color scale is used to map values: the minimum value of each measure is mapped to violet, the maximum value to magenta, and six intermediate values are mapped into blue, cyan, green, yellow, orange, and red.

Distortion seems better than the mean curvature estimator, in adapting smoothly to describe surface shape. It is less sensitive to noise in the data, and more effective in enhancing concave and convex areas. Note that the pulley mesh is not a CAD model but the result of scanning a real piece; distortion performs better in enhancing parts where the surface become irregular after long use.

As expected, both measures give large value to convex parts (peaks / ridges) and small values to concave parts (pits / ravines) of the terrain.

\section{Concluding Remarks}

We have presented the notion of discrete distortion for triangulated surfaces. We showed that distortion can be used as a good estimator of mean curvature. We discussed some issues related with the classical definition of discrete mean curvature mostly used in literature, and we showed that discrete distortion is more reliable. We have presented experimental results to illustrate the behavior of distortion and compare it with a mostly used discrete mean curvature estimator.

Our algorithm for computing discrete distortion can be used to simplify the algorithm in 4] that evaluates the Connolly function. The properties of discrete distortion make it suitable for use in many applications, such as physics of particles and chemistry. We are currently working on an algorithm that optimizes 
a triangulation using distortion as a metric. The optimized triangulation can be used to prove some properties that we conjecture for chemical reactions. It can also be used to for surface smoothing as discussed in [5].

\section{Acknowledgments}

This work has been partially supported by the National Science Foundation under grant CCF-0541032 and by the MIUR-FIRB project SHALOM under contract number RBIN04HWR8.

\section{References}

1. Alboul, L., Echeverria, G., Rodrigues, M.A.: Discrete Curvatures and Gauss Maps for Polyhedral Surfaces. In: Workshop on Computational Geometry, The Netherlands (2005)

2. Akleman, E., Chen, J.: Practical Polygonal Mesh Modeling with Discrete GaussianBonnet Theorem. In: Proceedings of Geometry, Modeling and Processing, Pittsburg (2006)

3. Baues, O., Peyerimho, N.: Geodesics in non-positively curved plane tessellations. Advanced in Geometry 6, 243-263 (2006)

4. Cazals, F., Chazal, F., Lewiner, T.: Molecular Shape Analysis based upon the Morse-Smale Complex and the Connoly Function. In: Proceedings of SoCG 2003 (2003)

5. Dyn, N., Hormann, K., Sun-Jeong, K., Levin, D.: Optimizing 3D Triangulations Using Discrete Curvature Analysis. In: Oslo, T.L., Schumaker, L.L. (eds.) Mathematical Methods for Curves and Surfaces, pp. 135-146 (2000)

6. Eisenhart, L.P.: Riemannian geometry. Princeton Univ. Press, Princeton (1949)

7. Garimella, R.V., Swartz, B.K.: Curvature Estimation for Unstructured Triangulations of Surfaces. Los Alamos National Laboratory LA-03-8240 (2003)

8. Gatzke, T.D., Grimm, C.M.: Estimating Curvature on Triangular Meshes. International Journal on shape Modeling 12, 1-29 (2006)

9. Gray, A.: Modern Differential Geometry of Curves and Surfaces Introduction to Computer Graphics. CRC Press, Boca Raton (1993)

10. Mangan, A., Whitaker, R., Partitioning, R.: Partitioning 3D Surface Meshes Using Watershed Segmentation. IEEE Transaction on Visualization and Computer Graphics 5(4), 308-321 (1999)

11. Mesmoudi, M.M., De Floriani, L., Danovaro, E., Port, U.: Surface Segmentation through Concentrated Curvature. In: Proceedings of International Conference on Image Analysis and Processing, vol. 1, pp. 671-676. IEEE Computer Society, Los Alamitos (2007)

12. Mesmoudi, M.M., De Floriani, L., Port, U.: Distortion in Triangulated 3-Manifolds. Computer Graphics Forum (the International Journal of the EUROGRAPHICS Association) 27(5), 1333-1340 (2008)

13. Meyer, M., Desbrun, M., Schroder, P., Barr, A.: Discrete differential-geometry operator for triangulated 2-manifolds. In: Proceedings of VisMath 2002, Berlin, Germany (2002)

14. The area of a spherical triangle, Girard's Theorem, http://math.rice.edu/ pcmi/sphere/gos4.htm 
15. Rousseau, J.-J., Gibaud, A.: Cristallographie Géométrique et Radiocristallographie. Dunod edn. (2007)

16. Spivak, M.: A comprehensive introduction to differential Geometry. Houston Texas (1979)

17. Stokely, E.M., Wu, S.Y.: Surface parametrization and curvature measurement of arbitrary 3-d objects: Five pratical methods. In: IEEE Transactions on pattern analysis and machine Intelligence, pp. 833-839 (1992)

18. Troyanov, M.: Les Surfaces Euclidiennes à Singularités Coniques. L'enseignement Mathématique 32, 79-94 (1986)

19. Port, U.: Shape Segmentation Algorithms Based on Curvature. Master Thesis, University of Genova, Italy (2007) 